\title{
Stereotipo della seduzione e seduzione dello stereotipo in La bella di Lodi di Arbasino
}

Andrea Inglese

\section{OpenEdition}

\section{Journals}

Edizione digitale

URL: http://journals.openedition.org/cei/807

DOI: $10.4000 /$ cei.807

ISSN: 2260-779X

\section{Editore}

UGA Éditions/Université Grenoble Alpes

\section{Edizione cartacea}

Data di pubblicazione: 15 septembre 2006

Paginazione: 23-32

ISBN: 978-2-84310-086-4

ISSN: $1770-9571$

Notizia bibliografica digitale

Andrea Inglese, «Stereotipo della seduzione e seduzione dello stereotipo in La bella di Lodi di Arbasino», Cahiers d'études italiennes [Online], 5 | 2006, online dal 15 mars 2008, consultato il 27 mars 2021. URL: http://journals.openedition.org/cei/807 ; DOI: https://doi.org/10.4000/cei.807 


\section{STEREOTIPO DELLA SEDUZIONE E SEDUZIONE DELLO STEREOTIPO \\ IN LA BELLA DI LODI DI ARBASINO}

Andrea Inglese

Université Paris 3

Vi propongo una riflessione sul romanzo breve La bella di Lodi di Alberto Arbasino. L'intreccio del romanzo si sviluppa intorno ad una circostanza di seduzione apparentemente tipica, se situata nel contesto della società italiana dei primissimi anni Sessanta: Roberta, rampolla di una ricca famiglia di Lodi, viene attratta da Franco, giovane e squattrinato meccanico. La storia ripercorre le alterne vicende di questa attrazione, fino allo scioglimento felice e banale, che si conclude con il matrimonio tra i due, una mésalliance dal sapore ottocentesco. Il mio intento è quello di mettere in luce il particolare trattamento narrativo al quale Arbasino sottopone un soggetto tipicamente romanzesco come quello della seduzione. È indispensabile, allora, fornire qualche chiarimento concettuale, prima di accostare il testo che ci interessa.

La circostanza di seduzione, da cui prende spunto la trama della Bella di Lodi, si presenta ad Arbasino già caratterizzata, almeno in parte, da un punto di vista ideologico. In primo piano emerge un rapporto tra due individui non perfettamente simmetrico e paritario, in quanto uno dei due possiede il potere, la forza, le qualità necessarie ad attrarre a sé l'altro, caratterizzandosi come il "seduttore", e l'altro dei due subisce questa attrazione, ne è in qualche modo soggiogato, caratterizzandosi come il "sedotto". Benché questo rapporto riguardi la sfera dell'eros, ossia l'ambito irrazionale delle pulsioni, esso si lascia leggere immediatamente in termini di potere. Ed è intorno alla nozione di potere, che la circostanza di seduzione acquista un aspetto propriamente "romanzesco". Finché restiamo nell'ambito di una perfetta reciprocità dell'attrazione, la seduzione si pre- 
sta difficilmente ad un fecondo trattamento narrativo: la naturalità dell'evento lo renderebbe piatto, banale, a meno di non introdurre, nel bel mezzo dell'idillio, il classico ostacolo all'unione degli amanti. Saremmo, però, di fronte a quella tipologia di ostacolo esterno che corrisponde più all'universo della fiaba che a quella del romanzo. La circostanza scelta da Arbasino non è di questo tipo. Egli si rifa al paradigma del romanzo psicologico novecentesco, che considera l'idillio amoroso minacciato in prevalenza da ostacoli interni, sorgenti nella dinamica stessa della relazione. Infatti Roberta e Franco, per buona parte del romanzo, sono esclusi dalla sfera dell'idillio, in quanto non si stabilisce tra loro una trasparente reciprocità di sentimenti. L'ostacolo interno, a cui la loro relazione sembra andare incontro, è legato al pericolo che uno dei due amanti sovrapponga il freddo calcolo alla libera espressione dei desideri. D'altra parte, se questa eventualità manifesta l'aspetto più inquietante della seduzione, ne fa anche un rivelatore della complessità dell'esperienza erotica degli esseri umani.

Il sospetto turba il libero slancio di Roberta, che teme di scorgere nell'atteggiamento di Franco il calcolo del profittatore. Questi, infatti, si presenta all'inizio della vicenda con la maschera del seduttore senza scrupoli: attira a sé Roberta, quasi subito la porta a letto, dopodiché se ne scappa mentre lei dorme, derubandola di ori e denari. Quando Franco, qualche tempo dopo, ricompare all'improvviso nella sua vita, Roberta, temendo un nuovo inganno, passa all'attacco, e si pone lei stessa nell'attitudine del calcolo: approfitta dell'impeto passionale di lui, per tendergli una trappola. Lo fa arrestare dai carabinieri, là dove si sono dati appuntamento. A questo punto è Franco a sentirsi tradito, in quanto tornava da Roberta con le migliori intenzioni, avendo preso coscienza di essere rimasto segnato dall'incontro amoroso con lei. Il rapporto tra i due nasce, quindi, all'insegna di un'improvvisa attrazione reciproca, disturbata però da sospetti e diffidenze, che ne insidiano l'immagine idillica di simmetria e sincerità.

Arbasino imbastisce un intreccio su di una circostanza di seduzione, secondo la prospettiva seria, anti-idillica, dell'ostacolo interno. Quest'ultimo nasce dalle complicazioni psicologiche, ma anche di classe e di stile di vita, cui vanno incontro i due protagonisti, consumando la loro relazione erotica. Ma nel caso di Roberta e Franco, l'ostacolo interno si rivelerà frutto di un'apparenza erronea, che ha forse radici nella paura di amare di entrambi i protagonisti. Dopo aver annunciato le lotte e i conflitti insolubili della relazione asimmetrica, Arbasino riconduce l'intreccio del romanzo psicologico a quello della commedia, che estingue gli ostacoli 
sia interni che esterni e si conclude con le nozze degli amanti. Tale operazione trae la sua efficacia proprio dall'aver sollecitato nel lettore una certa aspettativa nei confronti della vicenda erotica, che viene poi smentita dallo svolgimento della narrazione.

Mi preme ora richiamare alcuni motivi ricorrenti, che la rappresentazione romanzesca della seduzione offre al lettore novecentesco e a cui Arbasino fa riferimento, nel costruire con voluta ambiguità la storia di Roberta e Franco. Chi possiede, per dote naturale o sociale, esercizio misurato o inganno, la forza di attrarre l'altro, possiede anche un potere sull'altro, almeno fintantoché non soggiace lui stesso alla legge dell'attrazione reciproca. L'asimmetria e la non reciprocità permettono al seduttore d'introdurre nel rapporto erotico non la violenza, ma il calcolo e la finzione, ossia la possibilità di manipolare l'altro a piacimento e con il suo consenso. Questa manipolazione può servire vari scopi ed essere esercitata da diverse tipologie di individui. Dall'Ottocento in poi, il seduttore ha abbandonato i panni aristocratici del libertino, per assumere spesso quelli del nullatenente, ambizioso e cinico, uomo o donna, che attraverso il suo potere di seduzione tenta di farsi strada nella vita. L'avventura erotica è, in questi casi, l'occasione di un riscatto sociale e di un rovesciamento dei ruoli: chi è socialmente più forte, per status, ricchezza e cultura, si può trovare in una posizione di debolezza, dipendenza e sottomissione di fronte all'amante.

Ma il gioco di potere tra seduttore e sedotto tende ulteriormente a complicarsi, ed ecco che il più debole pretende, a sua volta, di garantirsi il controllo del più forte, seppure in modi non di rado vani e destinati allo scacco. Si pensi a quello straordinario romanzo sul tentativo fallimentare di ristabilire un rapporto di forza a proprio favore nel rapporto erotico, che è rappresentato dalla Prisonnière di Proust. Attraverso il suo delirio di gelosia, il protagonista esprime il disperato sforzo di controllare Albertine, che è padrona assoluta dei suoi pensieri e dei suoi sentimenti. Si tratta di un'impresa immane e impossibile: il sedotto pensa di incatenare a sé il seduttore, agendo proprio sul punto di forza di quest'ultimo, ossia il suo interesse. In sintesi, scambiando denaro per amore, Marcel pensa, come innumerevoli altre vittime dello stesso errore, di ristabilire la simmetria perduta nella relazione con Albertine.

Negli anni Sessanta, in Italia, tutti questi elementi concorrono ancora a caratterizzare la circostanza di seduzione e le zone di rischio, di conflitto o di impasse esistenziale, a cui essa può condurre gli individui che ne sono 
coinvolti. Un romanzo che funge da paradigma nell'esplorazione delle minacce e delle ebbrezze connesse alla seduzione, nella sua forma di relazione asimmetrica insidiata da ostacoli interni, è Un amore di Dino Buzzati, pubblicato nel 1963. Costruito secondo criteri prevalentemente realistici, Un amore ci presenta la tormentata relazione erotica dell'architetto Dorigo, benestante e cinquantenne, con Laide, giovanissima prostituta senza scrupoli, sullo sfondo della Milano del boom economico. Laide concentra in sé tutti quegli attributi della seduttrice depravata, "laida" appunto, che una certa cultura patriarcale e borghese ha reso privilegio delle figure femminili. Un'ombra fantastica e diabolica si proietta su di lei, ma nel contempo la metropoli stessa con i suoi nuovi fermenti indica che la realtà tutta sta cambiando e che la depravazione ha assunto un carattere frequente e banale. Una quantità di fattori, quali un più diffuso benessere, il rilassamento dei costumi, la mobilità sociale e le nuove ambizioni hanno moltiplicato le occasioni di seduzione e i rischi ad esse connessi. La ripetizione su larga scala finisce per attenuare, quindi, quegli aspetti, positivi o negativi, che renderebbero eccezionali tali esperienze. Ciò non accade nel romanzo di Buzzati, che espressamente vuole salvaguardare da ogni rischio di banalizzazione il nucleo dell'esperienza erotica di Dorigo. I tormenti del protagonista, manipolato in modo spietato da Laide, costituiscono, alla fine, l'opportunità di un riscatto conoscitivo, che gli permette di penetrare la sostanza della propria personalità e, più in generale, delle relazioni umane.

Non si tratta solo della scoperta, attraverso le dolorose tappe di una progressiva disillusione, di quali reali rapporti di forza entrassero nel legame erotico, volgendolo a proprio sfavore. La circostanza di seduzione ha infatti rivelato al protagonista, in vari momenti della sua vicenda, delle realtà che gli erano fino ad allora nascoste. Ecco un esempio, tratto da uno dei monologhi interiori di Dorigo:

Lui tartufo come tutti gli altri e adesso si accorge che cosa è la donna di importante per un uomo adesso si accorge come una bella ragazza può essere desiderata dai maschi adesso pensa e ripensa a quanto è falso il mondo che fa finta che non esistano i desideri carnali e non ne parla mentre in realtà ciascun uomo basta che sia sincero se incontri una ragazza anche per la strada una ragazza sconosciuta immediatamente pensa una cosa sola: è desiderabile? mi piacerebbe andarci a letto? ${ }^{1}$

Questa citazione ci permette di sollevare un'altra questione di carattere generale, che riguarda il modo in cui la circostanza di seduzione si presta

1. Buzzati D., Un amore, Milano, Mondadori, 1976 [1963], p. 171. 
non solo ad uno sviluppo narrativo, ma permette al romanziere di investire l'esperienza erotica di idee e valori secondo un'ottica spiccatamente personale, discostandosi da una valutazione più corrente e condivisa. In alcuni casi, narrare di un rapporto di seduzione significa penetrare la sfera dell'eros, per coglierne delle verità sulla condizione umana. La circostanza di seduzione diviene un pretesto per analizzare il comportamento erotico, e da questa analisi lo scrittore può trarre verità ignorate o dissimulate dall'opinione comune. Nell'esempio fornito da Buzzati, ciò che conta non è tanto il contenuto specifico della sua scoperta (il desiderio erotico sottende sempre e comunque ogni relazione tra uomo e donna), ma la pretesa che tale contenuto smascheri in qualche modo "la falsità del mondo" e l'“ipocrisia" del protagonista.

Parlerò di investimento ideologico dell'eros, per definire il tentativo di utilizzare l'esperienza erotica come chiave di lettura dell'intera esistenza umana. Narrare una circostanza di seduzione significa, allora, non solo disporre di una serie di azioni che coinvolgono una coppia di personaggi e che possono essere sviluppate in diverse direzioni, all'interno di un quadro di possibilità che vanno dalla reciprocità dell'attrazione perfetta e felice alla non reciprocità più spietata e funesta. Narrare la seduzione significa anche poter utilizzare certi fatti dell'eros come un argomento, per difendere o attaccare determinate idee sull'uomo o sulla società.

Queste premesse sono necessarie, perché vorrei mostrare come La bella di Lodi sia un romanzo che ha per soggetto una circostanza di seduzione, ma si caratterizza per un ostentato disinvestimento ideologico nei confronti dell'esperienza erotica. Tale disinvestimento ideologico, di cui chiariremo la natura da un punto di vista compositivo e stilistico, ha una portata polemica e, lungi dall'essere una restituzione del fatto erotico a una sua opacità e immediatezza originaria, si pone invece come critica dell'ideologia, nel solco del romanzo d'idee prediletto da Arbasino.

Ma vediamo brevemente la genesi del romanzo. La vicenda di Roberta e Franco ha come sfondo l'Italia del boom economico dei primissimi anni Sessanta. La bella di Lodi, infatti, appare inizialmente in forma di racconto nel 1961 sul "Mondo". Nel 1963, dal racconto è tratto il film omonimo di Mario Missiroli, alla cui sceneggiatura partecipa Arbasino. A distanza di circa dieci anni, La Bella di Lodi diviene un romanzo breve ed è pubblicato nel 1972. Racconto e sceneggiatura nascevano a margine di Fratelli d'Italia, la cui prima edizione è del 1963. Ora, l'interesse per La bella di Lodi è motivato proprio dal significato che assume l'operazione di riscrit- 
tura, che nell'opera arbasiniana è sempre un fatto denso di conseguenze. Si è detto, ad esempio, che Arbasino, riprendendo e sviluppando lo schema originario dell'avventura di Roberta e Franco (Giulia e Marcello, nel racconto del 1961), scrive in realtà contro se stesso, ossia non crede più nella storia che racconta ${ }^{2}$. Da tale premessa scaturirebbe una struttura narrativa dallo svolgimento abbastanza lineare, ma appositamente scucita, approssimativa negli indizi temporali e spaziali, frantumata ulteriormente grazie al procedimento dell'isolamento tipografico di brevi o brevissimi paragrafi tra ampi spazi bianchi.

Lo stile è vistosamente discontinuo, ma non nella direzione dell'eterogeneità sistematica del pastiche, praticato con oltranzismo nel precedente romanzo del 1969 Super-Eliogabalo. Nella Bella di Lodi, l'effetto è quello piuttosto di un'indecisione, per cui rivivono, allo stato di abbozzo o di sintetica esposizione, alcune costanti tecniche della scrittura di Arbasino, ma distribuendosi arbitrariamente nei diversi capitoli. Tornano insomma tutte le forme di riscrittura: dal rifacimento serio, à la manière di Balzac o Manzoni, al pastiche satirico, con gli accumuli di merci o reminiscenze Kitsch; dalla parodia delle conversazioni mondane alle tecniche del montaggio dadaista. Ma tutti questi procedimenti non rompono, come accadeva in Super-Eliogabalo, la continuità narrativa né esplodono attraverso le procedure di accumulazione e di amplificazione proprie del romanzosaggio, come accadeva in Fratelli d'Italia.

In Super-Eliogabalo, l'uso della citazione e del rifacimento hanno come scopo di rendere del tutto obsoleta ogni forma d'intreccio. Siamo al di là della dicotomia tra intreccio tradizionale, "naturalistico", incentrato sui fatti e sulla psicologia dei personaggi, e intreccio sperimentale, basato sulla stilizzazione dei caratteri sociali, sulle idee e sulla conversazione. Una tale opposizione interessava ancora l'Arbasino di Certi romanzi del 1964. Ora il plot di Artaud diventa un puro pretesto per incursioni caricaturali e innesti anomali, per associazioni arbitrarie di stili e di situazioni. Ma se l'ambizione è quella della distruzione dell'intreccio in quanto tale, attraverso una denigrazione e una deformazione protratte fin nelle sue più infime componenti, il risultato non appare privo di ambiguità. La realizzazione del libro sul niente, costruito per svuotamento di significati e loro

2. Vedi, ad esempio, le osservazioni di Carlo Bo: "il lettore è affascinato dalla capacità di Arbasino di gettarsi in una storia, senza nessun impaccio. Sennonché, poco dopo, è costretto a registrare una specie di delusione: innanzitutto lo scrittore non crede alla storia che ci vuole raccontare, spesso arriva addirittura a segnare un grosso margine di sfiducia nel "fatto"”. Bo C., Un Cocteau all'italiana, in "Corriere della sera", 27 aprile 1972, ora in Riga. Alberto Arbasino, a cura di M. Belpoliti e E. Grazioli, Milano, Marcos y Marcos, 2001. 
successiva equivalenza, è frenata nel suo procedere da controspinte improvvise. E Renato Rinaldi a farlo notare, in un saggio del 1985. La riscrittura di Arbasino, che dovrebbe ridicolizzare e smontare il testo di partenza, si trova spesso ad aderire, nel tono serio e nella prospettiva ideologia, al modello artaudiano. "Perciò - fa notare Rinaldi - il testo-fonte e il testo nuovo tendono spesso ad avvicinarsi, in nome di una serietà e di una profondità comuni”. ${ }^{3}$

Il riferimento a Super-Eliogabalo ci permette di chiarire un punto. Anche laddove l'esperimento letterario di Arbasino si fa più programmatico e radicale, mettendo in crisi ogni forma di rappresentazione romanzesca ereditata, non è assente l'investimento ideologico dell'eros. Esso emerge in diversi brani del romanzo, laddove il vitalismo erotico e lo spirito di trasgressione, che già animavano situazioni e personaggi di Fratelli d'Italia, assurgono a dottrina vera e propria. Cito da Super-Eliogabalo:

Eliogabalo si mette invece [...] alla testa di ogni gioventù che rifiuta il goal dell'efficienza aziendale come ideale spirituale individuale, e preferisce dipingersi la faccia e il corpo $[. .$.$] assorbire sostanze evasive e sognanti, esplorare la possibilità d'una sessua-$ lità di gruppo e di pelle... ${ }^{4}$

Un tale atteggiamento non può stupirci. Per Arbasino, e non solo per lui, gli anni Sessanta hanno rappresentato, in Italia, l'occasione per il legittimarsi sempre maggiore di un'ideologia di minoranza, incentrata sul tema della liberazione sessuale. Questa almeno è la nostra percezione retrospettiva. Noi sappiamo, oggi, che il boom economico e la diffusione su larga scala dei beni di consumo ha favorito anche la caduta di alcuni tabù sessuali, ancora ben radicati, alla fine degli anni Cinquanta, nella società italiana. Ma per molti artisti e scrittori questo nesso non appariva così scontato. Si pensi a certe pagine di un altro importante libro degli anni Sessanta, La vita agra di Luciano Bianciardi, pubblicato nel 1962. Bianciardi oppone di continuo la sessualità alienata o pubblicitaria alla sessualità sana ed elementare. La sessualità è divenuta, secondo lui, "il segno, l'ideogramma, il paradigma, il facsimile", 5 ossia tutto "simbolo" e niente "cosa". A questa sessualità parassitata dalle esigenze del consumismo, nuova autorità sui corpi più subdola di quella religiosa, Bianciardi oppone una sua visione autentica dell'esperienza erotica: "Ma io so che la noia finirebbe nell'attimo in cui si ristabilisse la natura veridica del coito.

3. Rinaldi R., Il romanzo come deformazione. Autonomia ed eredità gaddiana in Mastronardi, Bianciardi, Testori, Arbasino, Milano, Mursia, 1985, p. 228.

4. Arbasino A., Super-Eliogabalo, Milano, Adelphi, 2001 [1969], p. 49.

5. Bianciardi L., La vita agra, Milano, Rizzoli, 1998 [1962], p. 63. 
Lo so, finirebbe anche la civiltà moderna, perché il coito veridico non è spinta ad alcunché, si esaurisce in sé medesimo e, in ipotesi estrema, esaurisce chi lo compie" (La vita argra, p. 65).

Il paradosso è qui evidente: Bianciardi, nell'atto stesso di separare il sesso reale dal suo simbolo parassitario, investe a sua volta l'eros di valori e idee alternativi. Sostituisce un simbolo con un altro, il discorso ideologico di maggioranza con il discorso ideologico di minoranza. Nonostante $\mathrm{La}$ vita agra abbia pochi punti in comune con $U n$ amore, il romanzo di Buzzati quasi coevo, in entrambi gli autori emerge l'opposizione tra un'esperienza erotica autentica e personale, da un lato, e una alienata e di massa, dall'altro. Lo spiritualista Buzzati individua nell'eros un'esperienza quasi iniziatica e portatrice di una conoscenza superiore dell'uomo. Il materialista Bianciardi vede nell'eros la possibilità di un riconoscimento della propria autonomia individuale nei confronti dei poteri impersonali e manipolatori delle società avanzate. In entrambi i casi, ad un'ideologia dell'eros, quella dominante, se ne contrappone un'altra, elitaria e sovversiva.

Questo atteggiamento diventa esplicito in Super-Eliogabalo, che scritto nel 1968, si propone come consapevole manifesto "dell'Immaginazione contro ogni oppressione razionalistica e scientifica". Di questo manifesto, fa parte anche la celebrazione della corporeità e della sessualità liberata, proprio nel momento in cui tali temi, pur rimanendo legati al movimento della contestazione studentesca, si diffondevano grazie ad essa in sfere sempre più ampie della società e del dibattito pubblico.

Il romanzo La bella di Lodi non è allora soltanto riscrittura dell'omonimo racconto apparso nel 1961. Esso costituisce una riscrittura rivolta a quelle rappresentazioni dell'eros che da Buzzati e Bianciardi, all'inizio degli anni Sessanta, hanno condotto agli esiti militanti o di fiancheggiamento, di cui è protagonista Arbasino stesso con Super-Eliogabalo. In altri termini, con il suo nuovo romanzo Arbasino non contrappone più un'ideologia della sessualità ad un'altra. Si è infatti reso conto che, da una parte della barricata come dall'altra, il discorso, la dottrina, l'investimento ideologico finiscono per parassitare l'esperienza erotica, caricandola di promesse, di significati e di responsabilità, che ne snaturano il carattere e tendono a regolamentarla, a uniformarla, sia in direzione regressiva che trasgressiva.

In primo luogo, abbiamo visto come l'intreccio della Bella di Lodi sia costruito per disinnescare ad una ad una tutte le possibili minacce, tutti gli ostacoli interni ed esterni, connessi alla circostanza di seduzione. Le complicazioni psicologiche si appianano così come i conflitti di classe: l'espe- 
rienza erotica non presenta rischi, ma nemmeno rivela aspetti profondi o segreti dell'essere umano. I personaggi, d'altra parte, mancano completamente di spessore psicologico e sono presentati nel loro più esteriore comportamento fisico e verbale. Cito un passaggio, che compendia, a livello microtestuale, l'andamento narrativo dell'intero romanzo:

Loro ballano insieme per tutta la sera, si siedono, s'alzano, camminano, bevono, sorridono, soprattutto parlano: allegramente, anche seriamente, in confidenza, con scioltezza, figurine in un paesaggio d'estate padana, colorata, abbastanza sudata, pianura verdissima con filari di viti e di pioppi, ville, prati, architetture nobilissime, nuove fabbriche a centinaia $\left[\ldots .{ }^{6}\right.$

Roberta e Franco, come gli altri personaggi secondari, scorrono in effetti come "figurine" su diversi sfondi. Tanto i loro gesti e le loro parole sono ordinari, prevedibili e banali, quanto gli sfondi sono un compendio del Kitsch d'interni e d'esterni dei tardi anni Sessanta. Lo scioglimento felice più che manifestare la vivacità della commedia suggerisce la meccanicità del cliché e dello stereotipo, secondo il modello di un "romanzo da cartolina” prodotto in serie. D'altra parte, la cartolina pervade il paragrafo conclusivo del libro, fungendo da metafora dello stereotipo a cui è assimilata l'intera narrazione. Leggo la frase iniziale del paragrafo: "In una Venezia cartolinesca piena di sole e di turisti, al loro balcone d'albergo sul Canal Grande, Roberta e Franco affacciati guardano le gondole e i vaporetti" (p. 167). Qualche riga dopo: "Hanno già finito di firmare tutte le cartoline". E l'ultima frase del romanzo: "E tutt'e due, sereni, si voltano sorridendo al fotografo per la cartolina-ricordo" (p. 168).

Sul piano dell'intreccio, La bella di Lodi rovescia la strategia di SuperEliogabalo, senza per questo rinunciare a nessuno dei procedimenti avanguardistici già utilizzati sul testo artaudiano. Solo che ora non sono più i "frammenti mobili" a prevalere sulla trama, disintegrandola, bensì è la trama che, nella sua linearità, ingloba e subordina i "frammenti mobili" della storia di Roberta e Franco. L'intreccio, in ciò che ha di banale e rassicurante, assorbe in sé, neutralizzandole, le pretese dei punti di vista eccentrici disseminati qua e là nel testo. Nessun materiale e nessun procedimento sembra più poter sfuggire alla forza catalizzante dello stereotipo. Ecco allora come si può leggere La bella di Lodi, come un Dictionnaire des idées reçues sulla circostanza di seduzione e, più in generale, sulle promesse o le minacce dell'esperienza erotica. È in quest'ottica che si deve considerare il disinvestimento ideologico di Arbasino nei confronti dell'eros, non

6. Arbasino A., La bella di Lodi, Milano, Adelphi, 2002 [1972], pp. 113-114. 
già perché sia possibile individuare una zona liberata, un comportamento sessuale al di là dell'ideologia, ma perché ormai il discorso sull'eros è luogo comune, precede l'esperienza stessa degli individui, la illumina e la plasma. I fronti ideologici ancora contrapposti durante gli anni Sessanta, paiono essere, all'inizio degli anni Settanta, sul punto di riunificarsi, e costituiscono un discorso condiviso: il sesso deve essere liberato. L'eccentrico è ormai chi dice il contrario, una minoranza reazionaria e oscurantista. La buona società tutta, con sfumature diverse, parla con una voce sola, la voce dello stereotipo.

Tre sono le caratteristiche principali dello stereotipo. ${ }^{7}$ In primo luogo, esso associa almeno due nozioni, in questo caso: sesso e libertà. In secondo luogo, una volta stabilita l'associazione, essa può ripetersi automaticamente in contesti sempre diversi. Infine, la ripetizione implica anche un obbligo: sesso e libertà devono andare assieme. Non vi è più margine per scelte individuali, eccentriche, impreviste.

Tutto il romanzo di Arbasino è dunque attraversato dagli stereotipi. Li ritroviamo nelle descrizioni del paesaggio o dell'abbigliamento dei personaggi. Affiorano nei discorsi e nelle azioni. Costituiscono l'armatura rigida che sottende la commedia apparentemente serena di Roberta e Franco. Ma la normatività dello stereotipo trionfa delle inquietudini relative all'eros. D'ora in poi, sembra dirci Arbasino, sulla seduzione e sull'eros niente più sorprese. Ed è senz' altro parte del fascino della narrazione arbasiniana il fatto che una vicenda di attrazione erotica sia immersa in un'atmosfera così ovattata, distratta, placata. Ma La bella di Lodi ci dice però anche qualcos'altro, alla fine. La percezione precisa che il decennio che si è appena aperto all'insegna della trasgressione, rischi invece il più grande intorpidimento del desiderio.

Concludo, con una citazione da Fantasmi italiani. Nel brano Il bla, Arbasino realizza una flaubertiana raccolta di luoghi comuni. Ed eccone uno che fa al caso nostro: "Corpo - Da quando ha le sue problematiche, che brutto scopare, compagni...”. ${ }^{8}$

7. Vedi Slakta D., Stéréotype: Sémiologie d'un concept in Le stéréotype. Crise et transformations, a cura di A. Goulet, Caen, Presses Universitaires de Caen, 1994, pp. 35-46.

8. Arbasino A., Fantasmi italiani, Roma, Cooperativa scrittori, 1977, p. 66. 